\title{
Building Reliable Student Status Management System with Block Chain
}

\author{
Qun Chen \\ School of Computer Technology \\ Zhuhai College of Jilin University \\ Zhuhai, China
}

\begin{abstract}
At present, education-related corruption and illegal profit-making are frequent and incessant in China. The root is the problems in management of student status, including information dispersion, unsafe security, difficult verification, easy manipulation and counterfeiting. To a certain extent, it has been causing social impetuosity and confusion. Reconstructing the student status management system with block chain can effectively block up the loophole and eliminate the chaos. By using chain-splitting technology, we organize block chains according to administrative affiliation, learning stage and session; Student status chains and roster chains are constructed respectively to form a flexible hierarchical architecture; Active chains and inactive history chains will be handled skillfully. Thus, the running load and the amount of data stored in the block chain nodes do not accumulate over time. What's more, security and privacy of information are protected by security mechanism and information encryption of block chain. Without changing administrative system and rules, we can comprehensively solve the problems of objectivity, integrity, safety, flexibility and availability of student status, and eliminate educational fraud as well as return to the essence of education. Then education can truly guide the healthy development of individuals, families, society and the country.
\end{abstract}

Keywords-block chain; student status; school roll; management system

\section{INTRODUCTION}

Our country attaches great importance to education. The Constitution stipulates that every citizen has the basic right to receive education. We have been implementing nine-year compulsory education since decades ago. As the expansion of college enrollment since 1999, China has gone through the stage of popularization of higher education, and has entered the stage of universalization. Nowadays, in order to realize the great rejuvenation of the Chinese nation, it is proposed to build an innovative society with education and talents as the guide; In order to develop, all places mostly value talents, and have launched various policies of preferential treatment for talents, robbing them crazily; And families generally regard children's education as the top priority, while striving for good schools and attending various training and counseling classes on a daily basis is a lively scene in full swing in China.

Education is not only related to the prosperity or decline of the national fate, and the future of the nation, but also the key to individual destiny. Individual education experience and quality are measured on the basis of their status as a student.
However, under the current conditions of school roll management, the educational situation in China is worrying. Utilitarian and impetuous attitude for education are prominent in China. The gold content of diplomas is getting lower. On the one hand, the employment situation of university graduates is grim; on the other hand, it is difficult for employers to find suitable talents. Social success mode goes against successful learning mode. The problems behind these dilemmas are: The state can not get to know the situation of national educational resources, student resources and teaching and learning performance timely, objectively, accurately or comprehensively; Education inequality is still a major problem, because of not only the uneven allocation of educational resources, but also a variety of hateful educational corruption such as impersonating for school, immigrating for college entrance examination, obtaining admission qualification through the back door, falsifying papers and falsifying academic credentials, and various disgusting illegal acts surrounding the education industry such as manufacturing and selling fake diplomas and establishment of bogus colleges, which is difficult to eradicate; The quality of education has not been essentialy improved, which shows that students generally feel confused and are in a kind of Buddhist-style, being indifferent to everything and not eager to learn and make progress.

At present, the students' status in China is managed separately by various schools or local educational administration departments. Whether it is in the form of paper files or electronic files managed by computers, administrators have authority and convenient conditions to manipulate them. There is also the possibility of loss or damage due to improper safekeeping or other objective reasons. It is therefore unsafe. In addition, the school roll data form information islands, which can not be real-time interconnected, so it is actually difficult to verify. This makes people with bad intentions have chance to drill and make improper profits by making fakes, which is the root of the current educational chaos in China.

The emergence of block chain technology provides an effective technical means to solve the shortcomings of the student status management system. Based on block chain technology, this paper puts forward a solution to reconstruct the management system of Chinese students' status, which will comprehensively solve the problems of objectivity, integrity, safety, flexibility and availability of students' status without changing the administrative system and rules. Thus brings possibilities to eliminate educational fraud and get returning to 
the essence of education, so that education can truly guide individuals, families, society and the country.

\section{WHAT IS BLOCK CHAIN}

Block chain is a very powerful and revolutionary Internet technology developed in the past ten years. It can be used to build a self-operating social trust network independent of third parties, and promote the whole society to start value interconnection. Block chain technology is a new decentralized infrastructure and distributed computing paradigm, which uses encrypted chained- block structure to verify and store data, distributed node consensus algorithm to generate and update data, and intelligent contract to program and manipulate data. Block chain is characterized by decentralization, non-tampering, non-forgery, verifiability and anonymity. Since its introduction in 2008, block chain technology has gone through three stages: 1.0 stage (digital currency), 2.0 stage (digital assets and intelligent contracts), and 3.0 stage (intelligent society). At present, in the financial, telecommunications, medical and industrial automation industries, block chain technology plays a very important and key role as a basic supporting platform. For example, BAAS platform based on block chain technology serves various scenarios of intelligent application of financial business, intelligent detection scenarios of industrial automation, whole life cycle of drug development, food traceability, etc.

Block chains are trust-building tools that improve and enhance the flow, maintenance and use of data through distributed IT architecture, and further bring value in business and social governance. At the technical level, the distributed IT architecture of block chains has the characteristics of decentralization, transparency, consistency and strong dependence on cryptography; On the basis of these characteristics, on the data level, block chains can achieve data consistency on the basis of multi-party consensus, prevent data tampering, and can also trace the whole process of data-based application. At the business level, block chains can realize intelligent contracts of automated execution, and bring the value of business process optimization, mode innovation and new format cultivation in many industries. On this basis, it provides a new method for the wide participation of social governance, transparency and credibility, free sharing and auxiliary supervision.

\section{New Solution OF Student Status Management SYSTEM}

\section{A. Hierarchical Architecture}

At present, educational management system in China is divided into four levels, namely, national level, province or ministry level, prefecture or city level, and district or county level. The national level is managed by the Ministry of Education, which is the highest administrative organ for education management; Each province is responsible for the management of its subordinate prefectural or city education administration departments, as well as colleges and universities within its jurisdiction.; Each prefecture or city is responsible for the management of its subordinate district or county education administration departments, as well as the high schools within its jurisdiction; And each district or county is responsible for the specific management of compulsory education schools and kindergartens within its jurisdiction. There are provincial and ministrial colleges and universities, which are managed by the corresponding province or national ministry respectively. National universities are directly managed by the Ministry of Education. Students can only take the college entrance examination in the provinces where their household registration places are. And through selection examinations, students can enter high schools in their cities, junior middle schools in their districts or counties, and primary schools or public kindergartens near their household registration places.

Commonly, the student status management system needs to run continuously, while new data are constantly generated and old data must be preserved permanently. But in a block chain system, data stored in blocks can not be modified or deleted to ensure that the data is real and credible. Considering these technical characteristics and the existing education management system in China, the national student status management system based on block chain is designed as a hierarchical architecture as shown in Figure 1.

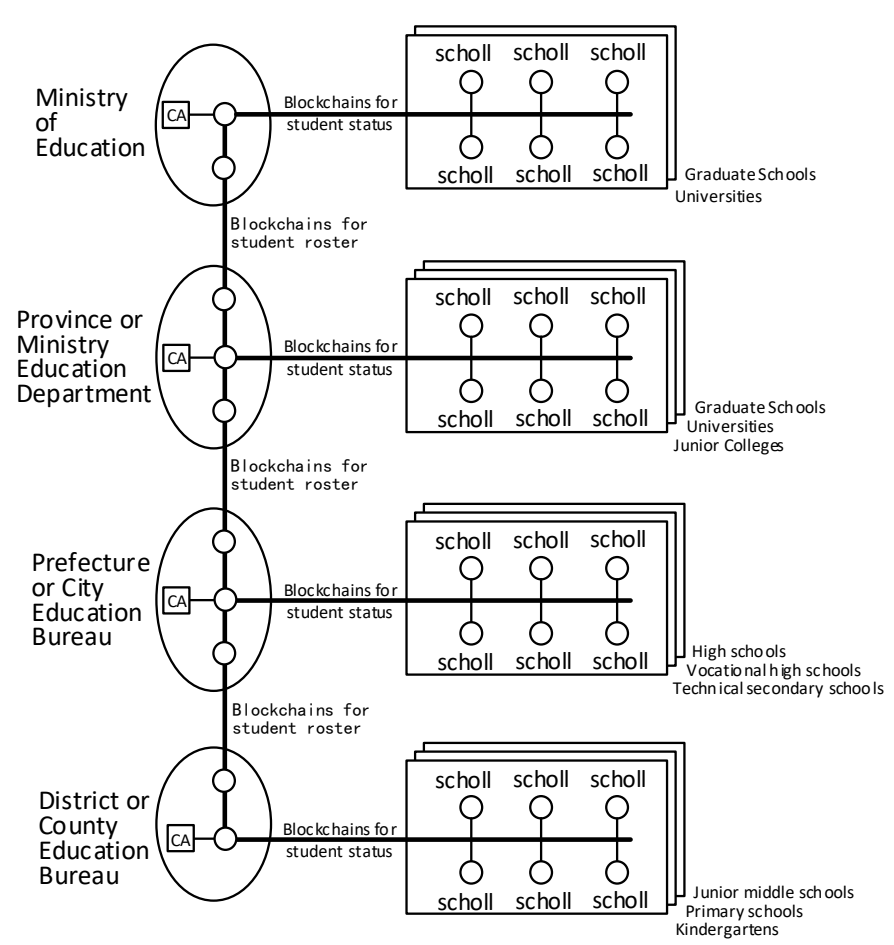

Fig. 1. Hierarchical architecture of student status management system based on block chain.

Set up block chain based student status management subsystem respectively in each district or county, prefecture or city, province or ministry (that ministry which manages universities) and the Ministry of Education. These four levels are interconnected according to the relationship of superiors and subordinates, thus constituting a new national integrated student status management system. 


\section{1) District or County Level}

In a district or county, all kindergartens and their (district or county level) superior management department are organized into one group, all primary schools and their (district or county level) superior management department are organized into another group, and all junior middle schools and their (district or county level) superior management department are organized into a third group. Within each group, the student status data of the same session of students in all schools are stored in the same independent block chain, which is called the student status chain. There are 13 active student status chains in a district or county. Among them, for kindergarten there are four active student status chains, which correspond to large class, middle class, small class and baby class; For primary school there are six active student status chains, which correspond to grade 1 to grade 6; For junior middle school there are three active student status chains, which correspond to grade 1 to grade 3 , respectively. In this way, for the students who have graduated from this learning stage (referring to kindergarten, elementary school or junior middle school), their student status chain of this stage will become an inactive chain, being archived and thus saving the storage space so as to avoid the infinite expansion of data on the working computers. When it is necessary to query the student status data of a graduated student, it can be realized by importing the archived data of that inactive chain into the working computer of the block chain sub-system.

How can we know in which student status chain the status data of a certain student is? It is unrealistic to go through the chains of all the active and the inactive historical, just as it is difficult to find a needle in a haystack. The solution is to set up a new roster chain besides the student status chain. At the prefecture or city level, all education bureaus of subordinate districts or counties and the relevant administrative department of this level shall be organized into a group. In this group, the student roster data of the same learning stage (referring to kindergarten, primary school or junior high school) in all schools are stored in the same independent block chain, which is called the student roster chain. In the student roster chain, the name (or the number) of the student status chain at each learning stage (referring to kindergarten, primary school or junior high school) is registered for each student. In this way, the name (or the number) of the corresponding student status chain can be obtained by searching the student roster chain first, and then the student status data of anybody at any stage can be obtained by this method.

\section{2) Prefecture or City Level}

There are three kinds of student roster chains maintained in a prefecture or city, corresponding to kindergarten, primary school and junior middle school. In a prefecture or city, all kindergarten students' roster data are registered in one roster chain, all primary school students' roster data are registered in another roster chain, and all junior middle school students roster data are registered in a third roster chain. With the admission of freshmen one session after another, the data on a student roster chain will continue to expand. Considering the bearing capacity of computer storage space, the student roster chain can be split by time, for example by decade. When a student roster chain is full of students of every 10 years, the old roster chain will be closed and a new roster chain will be created. When the students on a closed roster chain have graduated from this learning stage, the roster chain will become inactive, being archived to make room for storage on the working computer.

On the same principle, in a prefecture or city, all high schools and their (prefecture or city level) superior management department are organized into one group, all technical secondary schools and their (prefecture or city level) superior management department are organized into another group, and all vocational high schools and their (prefecture or city level) superior management department are organized into a third group. Within each group, the student status data of the same session of students in all schools are stored in the same independent block chain, which constitutes a student status chain. For the students who have graduated from this learning stage (referring to high school, technical secondary school or vocational high school), their student status chain of this stage will become an inactive chain and will be archived.

\section{3) Provice or Ministry Level}

At the province level, all education bureaus of subordinate prefectures or cities and the relevant administrative department of this level shall be organized into a group. In this group, the student roster data of the same learning stage (referring to high school, technical secondary school or vocational high school) in all schools are stored in the same independent block chain, which constitutes a student roster chain. In a student roster chain, the name (or the number) of the student status chain for each student at each learning stage (referring to high school, technical secondary school or vocational high school) is registered. There are three kinds of student roster chains maintained in a province, corresponding to high school, technical secondary school and vocational high school. In a province, all high school students' roster data are registered in one roster chain, all technical secondary school students' roster data are registered in another roster chain, and all vocational high school students' roster data are registered in a third roster chain. A student roster chain can be split by time, for example by decade. When a student roster chain is full of students of every 10 years, the old roster chain will be closed and a new roster chain will be created. When the students on a closed roster chain have graduated from this learning stage, the roster chain willl become inactive and will be archived.

In a province or ministry, all junior colleges and their (province or ministry level) superior management department are organized into one group, all universities and their (province or ministry level) superior management department are organized into another group, all master cultivation agencies and their (province or ministry level) superior management department are organized into a third group, all doctor cultivation agencies and their (province or ministry level) superior management department are organized into a fourth group, and all postdoctor cultivation agencies and their (province or ministry level) superior management department are organized into a fifth group. Within each group, the student status data of the same session of students in all schools are stored in the same independent block chain, which constitutes a student status chain. For the students who have graduated from this learning stage (referring to junior college, univerity, master, 
doctor or postdoctor), their student status chain of this stage will become an inactive chain and will be archived.

\section{4) National Level}

At the national level, all provincial and ministrial (that ministries which manage universities, including the Ministry of Education) education management departments shall be organized into a group. In this group, the student roster data of the same learning stage (referring to junior college, univerity, master, doctor or postdoctor) in all schools are stored in the same independent block chain, which constitutes a student roster chain. In a student roster chain, the name (or the number) of the student status chain for each student at each learning stage (referring to junior college, univerity, master, doctor or postdoctor) is registered. There are five kinds of student roster chains, corresponding to junior college, univerity, master, doctor and postdoctor. All junior college students' roster data are registered in one roster chain, all univerity students' roster data are registered in another roster chain, all master students' roster data are registered in a third roster chain, all doctor students' roster data are registered in a fourth roster chain, and all postdoctor students' roster data are registered in a fifth roster chain. A student roster chain can be split by time, for example by decade. When a student roster chain is full of students of every 10 years, the old roster chain will be closed and a new roster chain will be created. When the students on a closed roster chain have graduated from this learning stage, the roster chain will become inactive and will be archived.

\section{5) Others}

There are three reasons for setting up the student roster chain at the upper level above the student status chain. One is that it is technically convenient to query data across student status chains. The other is that it meets the administrative requirement of reporting of student status information to higher education management department. The third is that the unit data of the roster chain is simple and the amount of data is small, so roster for more students can be centralized stored. At the school level, whenever a student changes his or her status, including naturalization and non-graduation leave (such as repeating, skipping or dropping out), the change information should be reported to the education management department at the direct superior level in order to modify the information of the corresponding student roster chain.

CA (Certificate Authority) center and consensus center for block chain subsystem are established respectively at the prefecture or city level, province or ministry level, and the Ministry of Education, to serve the student status chains and the student roster chains running within the jurisdiction.

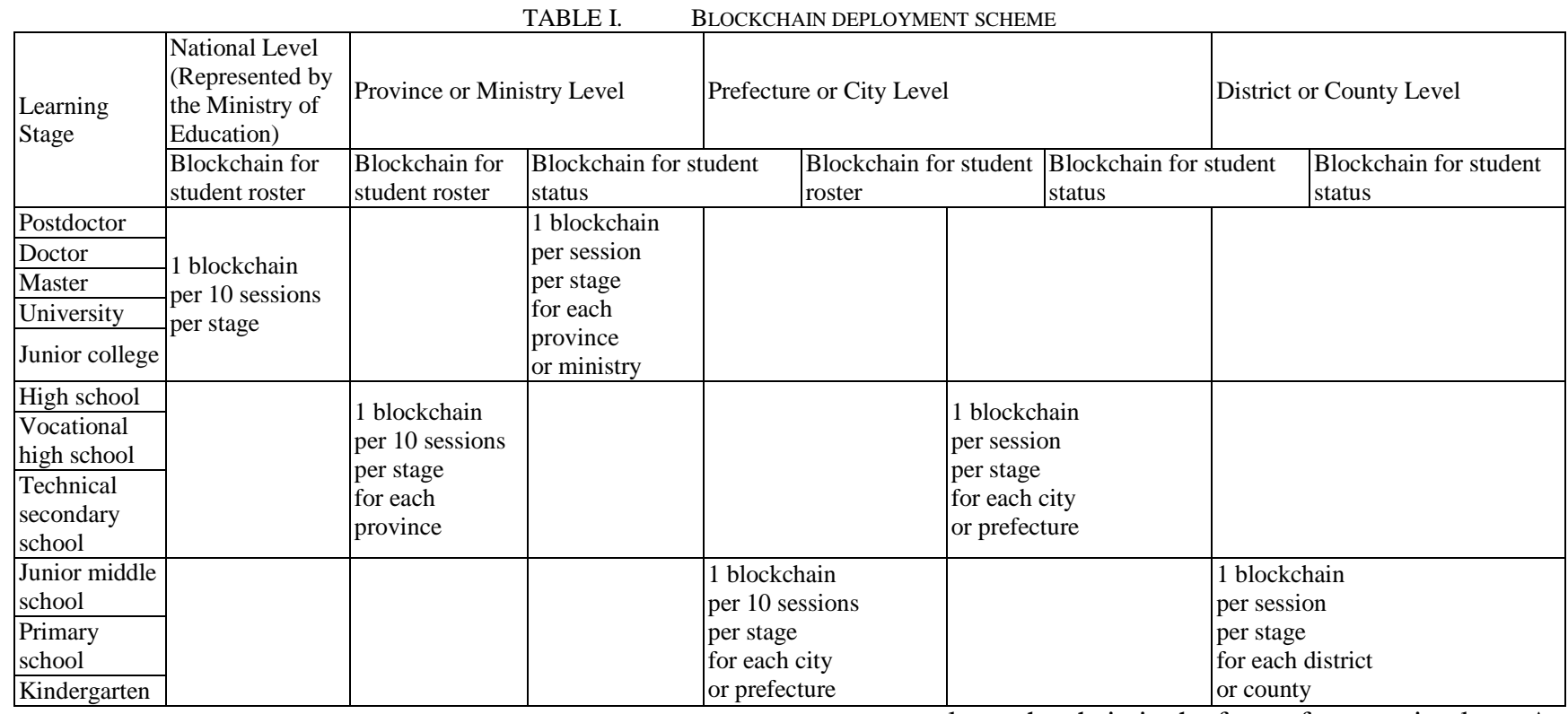

\section{B. Data Model}

There are three kinds of ledgers in each block chain subsystem. One is a chain consisting of blocks, which is used to store transaction logs. Once data is uploaded into the chain, it can not be added, modified or deleted, which is guaranteed by the inherent technical means of block chain technology. The other is a state database, which stores aggregated data generated from transaction logs, such as the balance of a financial account. And the third is an index database, which is used to assist in fast retrieval of transaction logs on the chain.

Schools usually taking semester as a cycle, submit students' status records, such as scores of subjects, rewards or punishments, to the block chain system, and keep them permanently on the chain in the form of transaction logs. At the same time, the intelligent contract on the block chain system collects the relevant information into the state database. The state database is usually a kind of key-value database. According to a key, such as ID number, or school number plus student number, the storage location of the corresponding keyvalue pair can be quickly found by a kind of query tree, as shown in Figure 2. The student status data or roster data of our system mentioned above can be stored in their respective state database by similar data structure. The data structure includes data head and data body. The data body is composed of several key-value pairs, such as "subject-score", while those key-value pairs are linked to each other by pointers. The data head contains header information and the count of key-value pairs in 
the data body, as well as a pointer linking to the first key-value pair in the data body.

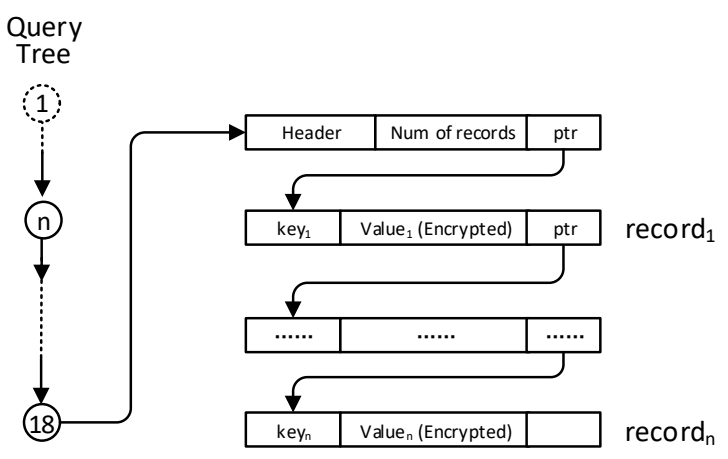

ID No.

Fig. 2. Data model of block chain state database

\section{Security and Privacy Management}

The recommended block chain technology is the third generation like Hyperledger Febric, which has excellent security and privacy protection mechanism and chain-splitting mechanism. The chain-splitting mechanism enables the abovementioned hierarchical architecture to be realized. In block chain technology, CA (Certificate Authority) and MSP (MenberShip Provider) services enable registered and authorized users to access the block chain system. Authorized access control and data confidentiality are realized through digital certificates, public-private secret Key pairs, digital signatures, HASH and data encryption and decryption. Block chain technology also achieves consensus among distributed nodes on the Internet through Byzantine fault tolerance or Kafka algorithm, so that the data on each computer node of the block chain remains consistent and correct.

Through the above mechanism of block chain technology, we can use intelligent contract to encrypt and store the data kept on the block chain ledgers, so that it can not be peeped or stolen by unauthorized users. Encrypted objects include the value of the key-value pair on the state database and the content of transaction log on the blocks of chain (not including the ownership information and "key" itself). Encryption and decryption are realized by intelligent contract, as shown in Figure 3. Data is encrypted when stored, but clear unencrypted information is used in application level.

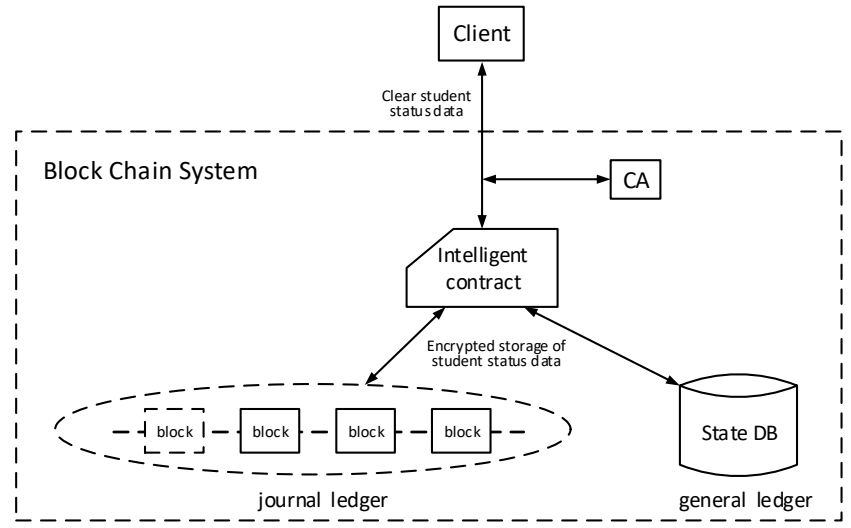

Fig. 3. Encrypted storage of student status data for security and privacy.
Authorized users at each level can only operate on data belonging to this level. For example, the administrator of a school can only access the student status chains of his or her school, and the administrator of a education management department can only access the student roster chains at the orresponding level.

\section{Storage Space and Scalability}

Using the chain-splitting mechanism of block chain technology, at each administrative level, we set up different chains of student status information for different sessions in different learning stages, and set up different chains of student roster information for different learning stages. The information of students who have not graduated from certain learning stage is on an activity chain. The information of students who have graduated from certain learning stage is on an inactive historical chain, and the chain can be archived and backed up to make room for storage on the working computer. This makes the storage space on a working computer almost constant, and there will be no data explosion and space overflow. Storing the information of students of different sessions in different chains not only facilitates the access to data, but also makes the system flexible and scalable, and ensures that the system can run steadily and continuously. When the system of education administration changes, or when a school is newly built, closed or merged, or the school's affiliation changes, our block chain based student status management system has enough flexibility to support such changes.

\section{CHARACTERISTICS}

\section{A. Scalability and flexibility}

Use block chain technology like Hyperledger Febric, which is building-block functional and supports multi-chain. Organize the student status chain according to the administrative affiliation, learning stage and session. Active chains run at block chain nodes, while non-active historical chains can be backed up offline. The quantity, scale and lifetime of chains are deterministic and limited. The quantity, computing power and storage space of block chain nodes can also be pre-determined. The architecture of the system can be flexibly assembled according to the application scenario, including the configuration of consensus module and security module. It can be implemented locally and interconnected to form a national unified system.

\section{B. Performance and availability}

The quantity and the load of block chain nodes are deterministic and stable, and are not subject to the impact of data or access surge over time. The student roster chain is separated from the student status chain, and the capacity of each chain is limited. So the enquiry of student status information becomes simple and efficient. 


\section{Security and Privacy}

Block chain technology ensures that data is not lost, damaged or forged, and is verifiable and traceable. Combining the advantages of block chain security mechanism and hierarchical management mode, as well as generating, auditing, maintaining and using student status data according to subordinate management, the scope of security domain is narrowed. By encrypted storage the value of key-value pairs and the associated content of transaction logs, the distributed block chain nodes will not leak data unrelated to themselves.

\section{Integrity and Reality}

The block chain technology involved is mature and reliable, and its function and performance can fully meet the needs. The technical model, logical rules and interconnection method of the system are simple and easy to operate. The system model is completely compatible with the existing organization and management models. On the same technical specifications, we can build a nationwide unified system of student status block chain from bottom to top, so that the information of student status can be fully integrated. It can be built on existing server devices and network facilities, with low additional cost.

\section{CONCLUSION}

Building a student status management system based on block chain can ensure the objective, real-time and permanent existence of the student status information for all people in the country. The scheme involves mature and reliable technology, and clear and simple rules, which is fully compatible with the real organization and management model and material conditions, without too much additional investment, and can be implemented step by step, with short construction cycle and quick results. In the process of implementation, it is necessary to carefully design specific technical specifications, including data structure, allocation of authority, encrypted objects and encryption methods, logical rules of intelligent contracts, strategies for backup and recovery of historical chains, and so on. Consideration should also be given to what security modules and consensus algorithms are used and at what level they are deployed.

\section{ACKNOWLEDGMENT}

\section{Corresponding author: Qun CHEN}

Correspondence: 519041, School of Computing in Chu Hai College of Higher Education, Jilin University located in Sanzao Town, Jinwan District, Zhuhai City, Guangdong Province.

\section{REFERENCES}

[1] Junfei HUANG and Jie LIU. 2018. Review of Block Chain Technology. J. Journal of Beijing University of Posts and Telecommunications. 2. (Feb. 2018), 1-8.

[2] Qifeng SHAO, Chengqing JIN, Zhao ZHANG, Weining QIAN and Zhou Aoying. 2018. Block Chain Technology: Architecture and Progress. J. Chinese Journal of Computers. 5. (May. 2018), 969-988
[3] Qifeng SHAO, Zhao ZHANG, Yanchao ZHU and Aoying ZHOU. 2019. Overview of Enterprise Block Chain Technology. J. Journal of Software. 4 (Apr. 2019). DOI: 10.13328/j.cnki.jos.005775. http://kns.cnki.net/kcms/detail/11.2560.TP.20190409.1732.004. html

[4] Yong YUAN, Xiaochun NI, Shuai ZENG and Feiyue WANG. 2018. Current Situation and Prospect of Block Chain Consensus Algorithms. J. Acta Automatica Sinica. 11. (Nov. 2018), 20112022.

[5] Haiwu HE, An YAN and Zehua CHEN. 2018. Overview of Intelligent Contract Technology and Application Based on Block Chain. J. Journal of Computer Research and Development. 11 (Nov. 2018), 2452-2466.

[6] Chen PAN, Zhiqiang LIU and Zhenlongyu LIU. 2018. Research on Block Chain Scalability: Problems and Methods. J. Journal of Computer Research and Development. 10. (Oct. 2018), 20992110.

[7] Xuan HAN, Yong YUAN and Feiyue WANG. 2019. Block Chain Security: Current Situation and Prospect. J. Acta Automatica Sinica. 1.(Jan. 2019), 206-225.

[8] Xian ZHANG, Yuzhao JIANG and Ying YAN. 2017. Overview of Block Chain Privacy Technology. J. Journal of Information Security Research. 11 (Nov. 2017), 981-989.

[9] Lihuang ZHU, Feng GAO, Meng SHEN, Yandong LI, etc.. 2017. Review of Block Chain Privacy Protection. J. Journal of Computer Research and Development. 10. (Otc. 2017), 21702186.

[10] Weili CHEN and Zibin ZHENG. 2018. Block Chain Data Analysis: Current Situation, Trends and Challenges. J. Journal of Computer Research and Development. 9. (Sept. 2018), 18531870.

[11] Weide CAI, Lian YU, Rong WANG, Na LIU and Enyan DENG. 2017. Research on Development Method of Application System Based on Block Chain. J. Journal of Software. 6 (Jun. 2017), 1474-1487.

[12] Tengfei XUE, Qunchao FU, Zhong WANG and Xinyan WANG 2017. Research on Medical Data Sharing Model Based on Block Chain. J. Acta Automatica Sinica. 9. (Sept. 2017), 1555-1562.

[13] Zhongzhen LI, Chao GAO, Min LIU, Chengqin DAI and Yi FAN. 2019. Student Status Management System Based on Block Chain Technology. J. Journal of Sichuan University(Natural Science Edition). 3. (Mar. 2019), 450-456.

[14] Lin ZHU and Yue LIU. 2019. Research on Management System of College Students'Daily Behavior Based on Block Chain Technology. J. Journal of Southwest Minzu University(Natural Science Edition). 3. (Mar. 2019), 290-295.

[15] Haibo TAN, Tong ZHOU, He ZHAO, etc.. 2019. Archives Data Protection and Sharing Method Based on Block Chain. J.

Journal of Software. 4. (Apr. 2019). DOI: 10.13328/j.cnki.jos.005770. http://www.cnki.com.cn/Article/CJFDTotalRJXB20190409000.htm 